\title{
The adaptation of trust behavior and the association with friendships in adolescents
}

H. Sijtsma ${ }^{1}$, N.C. Lee ${ }^{1}$, M.T.R. van Kesteren ${ }^{2}$, M. van Buuren ${ }^{1}$, B.R. Braams ${ }^{1}$, N.M. van Atteveldt $^{1}$, L. Krabbendam ${ }^{1}$

${ }^{1}$ Section of Clinical Developmental Psychology, Research Institute LEARN!, Institute of Brain and Behavior, Faculty of Behavioral and Movement Sciences, Vrije Universiteit Amsterdam

${ }^{2}$ Department of Educational and Family Studies, Institute of Brain and Behavior, Faculty of Behavioral and Movement Sciences, Vrije Universiteit Amsterdam

Corresponding author:

Hester Sijtsma

h.sijtsma@vu.nl 


\begin{abstract}
Being able to adapt one's trust behavior is essential for social relationships. In the current fMRI study, we first examined adolescents' ability to adapt trust behavior and the neural mechanisms of trust. Second, we examined developmental differences by comparing young and late adolescents. Third, we examined how positions in a friendship network, derived from social network analysis, relate to trust behavior in the late adolescent group. Seventeen young adolescents (Mage=12.6, $S D=0.9,10$ female) and 33 late adolescents (Mage $=17.2, S D=0.5,25$ female) played two trust games. Participants received a priori information suggesting that one partner was trustworthy and one partner untrustworthy. In reality, the behavior of both partners was programmed as trustworthy behavior. Results indicated that adolescents adapt their trust behavior when incorrect a priori information was provided, and developmental differences in this ability to adapt trust behavior were found. When incorrect a priori information was provided, late adolescents showed more dlPFC activity when receiving the partner's feedback compared to young adolescents. Furthermore, late adolescents with less central network positions were more adaptive in their trust behavior compared to late adolescents with more central positions. This study provides insight into how age and social relationships influence trust behavior during adolescence.
\end{abstract}

Keywords: a priori information, trust behavior, dlPFC, adolescence, social network analysis 


\section{Introduction}

Adolescence is a period of great social change. An important component of successful social relations is developing trust in others (van den Bos et al., 2010), a skill which improves with age (Fett et al., 2012; Sutter \& Kocher, 2007; van den Bos et al., 2010). Trust has been related to social relationships and social networks in companies and working life (Burt et al., 2013). A crucial aspect of the development of trust is the fine-tuning of trust behavior in response to the social context and to the trustworthiness of others (van den Bos et al., 2010). During adolescence and young adulthood, people learn to adapt their trust behavior in response to information they receive about their interaction partners (Delgado et al., 2005; Lee et al., 2016). Neuroimaging research has suggested that cognitive processes such as mentalizing, reward processes and conflict monitoring are involved in trust behavior (Bellucci et al., 2017; Fett et al., 2012; King-Casas et al., 2005). The first aim of the current study is to investigate how adolescents adapt their trust behavior and to investigate the neural mechanisms of trust. Second, we examined age-related changes in these processes. The third aim is to investigate for the group of late adolescents how social network positions in daily life are related to behavioral adaptation processes of trust and to the neural mechanisms of trust.

Trust behavior is often investigated using a trust game. The multi-round trust game simulates dynamic social interactions in which two players share money in multiple rounds on the basis of trust (Berg et al., 1995). In every round the participant (the investor) shares an amount of money with the partner (the trustee) which is then multiplied by a predetermined factor, often three. The partner decides whether or not to return an amount to the investor. Using the trust game, we can investigate baseline trust behavior, average trust behavior and the adaptation of trust behavior during the course of the game. 
The design of the trust game allows implementation of experimental manipulations, especially when a hypothetical partner is used. For example, a priori information on the trust behavior of the partner can be provided, which can be either in line or not in line with the actual behavior of the partner over the course of the game (Bailey et al., 2016; Fareri et al., 2012; Fouragnan et al., 2013; Lee et al., 2016; Van't Wout \& Sanfey, 2008; Wardle et al., 2013). This enables investigation of the relative influence of a priori information versus actual feedback during the game on trust behavior. When such information is not in line with actual behavior, for example if the partner is less trustworthy than the information suggests, the participant needs to recognize this discrepancy and adapt their own trust behavior.

Cognitive processes such as mentalizing, reward processes, cognitive control, and conflict monitoring processes are addressed during the trust game (Bellucci et al., 2017; Fett et al., 2012; King-Casas et al., 2005). Both players use mentalizing processes to evaluate the behavior and the intentions of the other player as they build a mental model of the trustworthiness of the other. Social reward learning processes play a role as it can be rewarding when someone trusts you, when your own trust behavior is reciprocated, or when the partner is more trustworthy than was expected. Conflict monitoring processes can be involved when the partner behaves untrustworthily while cooperation was expected, or when the partner's behavior is inconsistent compared to a priori information. Results from neuroimaging studies support the involvement of these processes. For example, previous studies have shown that areas such as the temporal-parietal junction (TPJ) and the medial prefrontal cortex (mPFC) are active during the trust game, and activity in these areas has been associated with mentalizing processes (Fett et al., 2012; Lemmers-Jansen et al., 2017; Van Den Bos et al., 2009, 2011). The dorsolateral prefrontal cortex (dlPFC) and the anterior cingulate cortex $(\mathrm{ACC})$ are related to conflict monitoring processes and are also found to be active 
during the trust game (Fett et al., 2012; Lemmers-Jansen et al., 2017; Van Den Bos et al., 2009). Increased activity in reward-related areas such as the caudate and insula has also been found in previous studies, both in games with and without a priori information about the interaction partner (Delgado et al., 2005; Fareri et al., 2012; Fett et al., 2012; Fouragnan et al., 2013; Lemmers-Jansen et al., 2017; Phan et al., 2010; Van Den Bos et al., 2009). For example, when participants have an expectation about the partner that is based on a priori information, caudate activity is reduced when processing the partner's response compared to situations in which there is no a priori information (Delgado et al., 2005; Fouragnan et al., 2013). Activity in the ventral striatum was not differently affected by a priori information compared to no a priori information (Fareri et al., 2012), although in another study, ventral striatum activity was increased when the participant was told the partner was trustworthy compared to when playing with a neutral partner (Phan et al., 2010).

Previous work suggests developmental effects in trust behavior when playing with a trustworthy partner. For example, late adolescents (Mage=17.5) are more able to adapt their behavior when a priori information is not in line with actual behavior compared to young adolescents (Mage=13.2) (Lee et al., 2016). Nevertheless, even in late adolescents (Mage=19.1) and young adults $(M a g e=26.6)$, a priori information which is discrepant with actual behavior continues to have an effect on trust behavior throughout a multi-round game (Delgado et al., 2005; Zarolia et al., 2017). Furthermore, age effects have also been found in the neural mechanisms of trust. A positive association between age and activity in TPJ, the posterior cingulate gyrus, the precuneus, the precentral gyri, the middle frontal gyri and the dlPFC was shown when playing a trust game (Fett et al., 2012, age 13-49; Lemmers-Jansen et al., 2017, age 16-27). A negative association between age and activity in the orbitofrontal cortex and the caudate nucleus, previously related to reward processes, and activity in the dorsomedial prefrontal cortex, previously related 
to uncertainty, was found (Fett et al., 2012; Volz et al., 2005). In a more recent study using a smaller age sample, no developmental effects in the neural mechanisms of trust were found (Lemmers-Jansen et al., 2019, age 13-19).

As trust is assumed to be essential in social relationships, an important question is to what extent behavior in the trust game is actually associated with social relationships in daily life. Social network analysis is a useful analysis technique to investigate these associations, as it is suitable to examine the different positions that individuals occupy within a social network (Robins, 2015). In initial research that was done in companies, it was shown that trust between people is higher in more dense social networks, and between people in embedded, close relationships, as people get to know each other well and untrustworthy behavior is more easily identified (Burt et al., 2013). Furthermore, people who report having more social relationships show more trust in others (Buskens, 1998). In another study, no association between social network positions and trust was reported but a positive relationship between the centrality of social network positions and prosocial behavior was found (van den Bos et al., 2018).

In the current study, we used the trust game to first investigate the ability of adolescents to adapt their trust behavior and to investigate the neural mechanisms of trust. Second, we examined whether the behavioral adaptation processes and the neural mechanisms of trust behavior differ between young adolescents (11-13) and late adolescents (16-18) during interactions with a preprogrammed partner who behaves differently than expected based on a priori information. The third aim was to investigate for the group of late adolescents how social network positions in daily life are related to behavioral adaptation processes of trust and to the neural mechanisms of trust during interactions with a preprogrammed partner who behaves differently than expected based on a priori information. To this end, participants played a trust game with two different partners, 
though the behavior of both partners was determined by the same computer algorithm that was modeled trustworthy behavior. Both games started with a priori information describing the behavior of the partner. One partner was suggested to be trustworthy (the 'consistent partner' because the a priori information and the trust behavior were both trustworthy) and the other partner was suggested to be untrustworthy (the 'inconsistent partner' because the a priori information described untrustworthy behavior but the behavior was trustworthy).

Concerning the first research aim, a greater adaption of adolescents' trust behavior, reflected in an increase of investments, was expected when interacting with the inconsistent partner compared to the consistent partner as the a priori information about the inconsistent partner is less in accordance with the actual trust behavior. During trust decisions with both partners, increased activity was expected in areas associated with mentalizing such as the TPJ and the amPFC when compared to the control trials. More activity in these mentalizing areas was expected during trust decisions with the inconsistent partner compared to the consistent partner, as one will think more about the partner's intentions when the a priori information and the behavior deviate. In comparison to control trials, we expected increased activity in both conditions in areas associated with conflict monitoring and reward processes (dlPFC, ACC, caudate, insula, ventral striatum) when receiving the partner's response. We expected more activity in these areas when interacting with the inconsistent partner compared to the consistent partner because the inconsistency between the a priori information and the actual behavior would create more conflict monitoring. For the second research aim on examining age differences during interactions in which a preprogrammed partner behaves differently than expected, we hypothesized the behavioral adaptation with the inconsistent partner to be greater for late adolescents compared to young adolescents. In line with the behavioral hypothesis, we expected the activity in mentalizing and conflict monitoring areas 
to be greater for late adolescents compared to young adolescents. For the third aim we examined for the late adolescent group, the effect of social network position on trust during interactions in which a preprogrammed partner behaves differently than expected. We hypothesized that late adolescents with more central network positions would adapt their trust behavior faster towards the inconsistent partner because of their many social relationships compared to late adolescents with less central network positions. This faster behavioral adaptation is expected to be associated with increased activity in areas related to mentalizing processes.

\section{Methods}

\section{Participants}

The study sample comprised a late adolescent group and a young adolescent group. The participants in the late adolescent group were recruited via a larger research project which took place at two high schools. This made it possible to collect social network data for this group. The participants were invited to additionally participate in the current fMRI study. The participants in the young adolescent group were recruited via an existing database. The participants signed up to this database individually and not via their schools, hence social network data was not collected in this group.

The late adolescent group comprised 39 adolescents (16-18 years old). Of the late adolescent group two participants were excluded due to too much head movement during fMRI scanning $(>3 \mathrm{~mm})$, three participants were excluded due to technical issues during scanning and one participant was excluded because the task instructions were not understood properly. Of the late adolescent group, 33 participants were included in the fMRI analyses $(M=17.2$ years old, 
$S D=0.5$, range $=16.1$ to $18.3,25$ female). We could not use the social network data of three participants as the participation rate within their school grade was too low to create reliable social network position (see social network positions) and for four participants the social network data was unavailable. So, 26 participants of the late adolescent group were included in the social network analyses $(M=17.2$ years old, $S D=0.5$, range $=16.1$ to $18.3,20$ female $)$.

The young adolescent group comprised 21 adolescents (11-13 years old). Of the young adolescent group one participant was excluded due to too much movement $(>3 \mathrm{~mm})$, two participants were excluded due to technical issues during scanning and one participant was excluded because the task instructions were not understood properly. Therefore, 17 participants from the young adolescent group were included in the analyses $(M=12.6$ years old, $S D=0.9$, range $=11.0$ to $13.7,10$ female). All participants received $€ 15$,- for participation and an additional monetary bonus based on their earnings during an arbitrary round of the trust game (approximately seven euros). The study was approved by the Scientific and Ethical Review Board of the Faculty of Behavioral and Movement Sciences of the Vrije Universiteit Amsterdam.

\section{Measures}

\section{Trust game and trust game questionnaire}

Participants played two trust games in the scanner (the order of the games was counterbalanced). Each game consisted of twenty randomized experimental trials and twelve control trials (see Figure 1). The behavior of both partners was determined by the same algorithm and modeled trustworthy behavior. An experimental trial started with a cue to invest, followed by the participant making an investment between $€ 0$,- and $€ 10$,- by pressing the index finger. The investment was 
multiplied by three. A waiting period followed and subsequently the returned amount of money was shown. A screen with a fixation cross followed and then the total earnings for that trial for each player were shown. The trial ended with a fixation cross. During control trials the participant selected a number rather than making an investment, but both types of trials had comparable visual input (see Figure 1). The trust game was presented in Presentation ${ }^{\circledR}$ software. The task was shown on a projection screen that participants could see through a mirror on the head coil. After the game, participants rated the partner on trustworthiness on a scale from 1 to 7 .

The participants were told to play a trust game with an age-matched partner. In reality, the behavior of the partner in both games was modeled by a computer algorithm. The algorithm modeled trustworthy behavior as the partner's return was never lower than the investment made by the participant. The partner's return was based on the trustor's investment multiplied by a predefined factor. At the start of each game, the factors were 1.0, 1.1, 1.2, 1.3, 1.4 and 1.5. All factors had an equal chance of $1 / 6^{\text {th }}$ to be selected and this was done at random. The value of the factors changed over the course of the game, based on the whether the trustor increased or decreased their investment compared to the previous trial. In case of an increase, all factors were increased with 0.05 . In case of a decrease, all factors decreased with 0.05 . The minimum value for the factors was 1.0 and the maximum value for the factors was 1.6. 


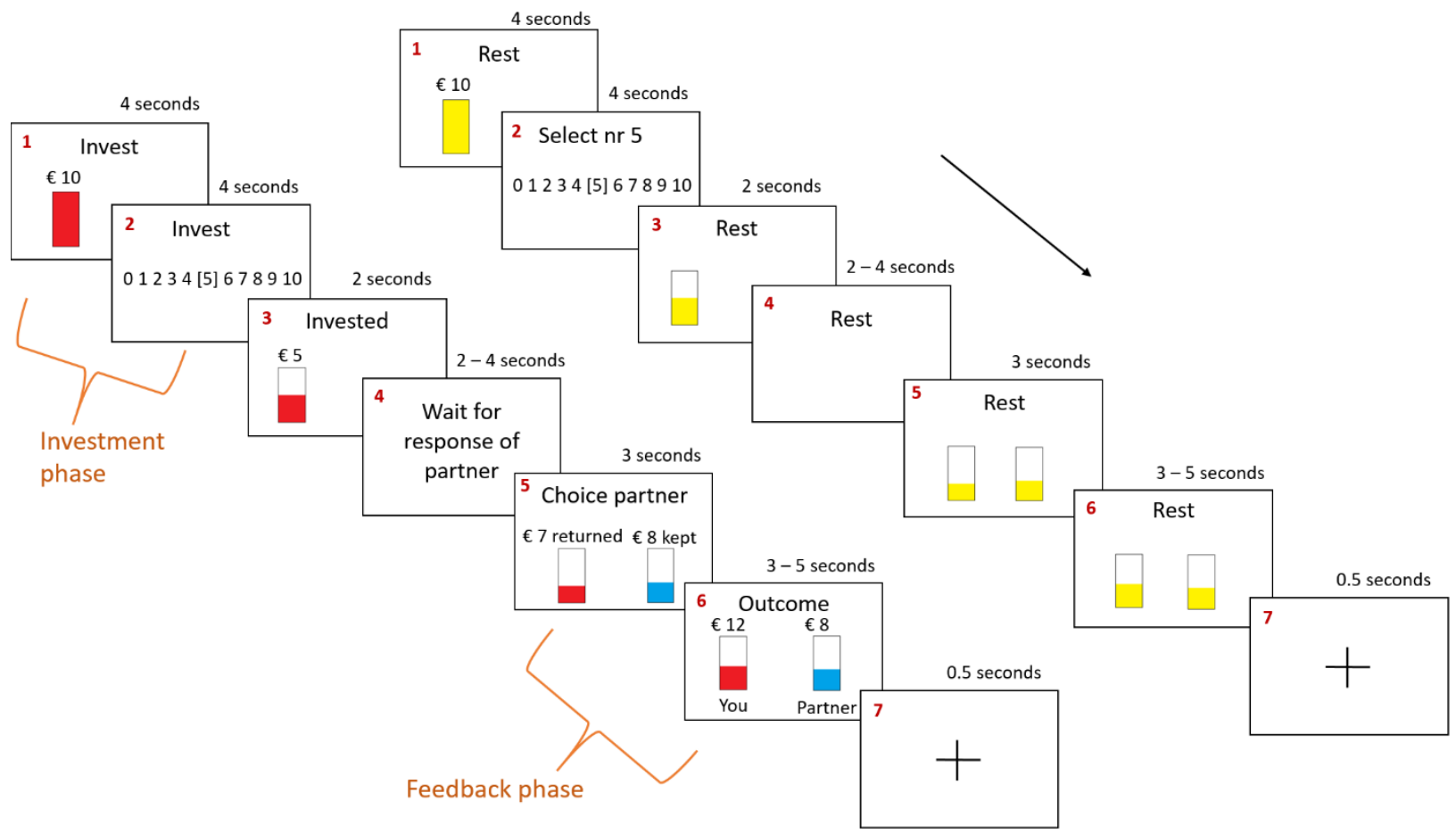

Figure 1. An experimental trial (left) and a control trial (right). Durations of the different events are indicated above the screenshots.

\section{Social network positions}

As mentioned above, the participants in the late adolescent group were recruited via a larger research project. As many pupils within the same grade participated in the larger research project, social network data was collected in the larger project and available for the current study. In the larger research project, a peer nomination questionnaire was administered to all pupils of the $11^{\text {th }}$ grade of a high school in two consecutive years $(n=127, n=111)$ and for the other high school this was done for one year $(n=96)$. A minimum of $75 \%$ of the pupils in a class or in a grade participating in the social network questionnaire is desirable to create reliable social friendship networks (Cillessen \& Marks, 2011; Marks et al., 2013). In one year $(n=111)$ the participation rate was 
below $75 \%$ so participants from this grade were excluded for the social network analyses. The participation rates in the other two grades were above $80 \%$.

To measure individual social network positions, the eigencentrality of each participant's position in a friendship network was calculated using the question 'Who are your friends?'. A maximum of 15 friends could be selected from a list of all pupils in the $11^{\text {th }}$ grade. The data was symmetrized according to the minimum procedure, meaning that a friendship was present when two pupils both selected each other as a friend (i.e. reciprocal friendships). The program UCINET was used to calculate the eigencentrality of each participant (Borgatti et al., 2002). This measure shows how central each person is within the network, where more reciprocal friendships relates to a higher eigencentrality score. The eigencentrality of the persons to which one is connected, is included in the calculation so that the more central your friends are, the more central you become (Robins, 2015). To control for differences in group sizes across different grades, we used the normalized eigencentrality calculated by UCINET.

\section{Procedure}

Consent for collection of the social network data from the late adolescents in the larger research project was given actively by the participants and passively by the parents. For the current fMRI study, all participants gave written informed consent (active) and in case the participant was under the age of 18, the parent/caregiver was also asked to give written informed consent (active). Participants were told that they would play a game with two same-aged peers who were participating in the current study at another imaging center. Participants played the role of the trustor and the peer played the role of the partner. First, the participant played three acquaintance rounds with each partner. In each round the participant made an investment but the response of the 
partner was not revealed yet. Next, we informed the participant about the behavior of the partner during the acquaintance rounds and then the first trust game started. Depending on the condition, the participant received the information 'the partner always returned more than the investments you made' (the consistent partner) or 'the partner never returned more than the investments you made' (the inconsistent partner). This information served as a priori information about the trustworthiness of the partner, though the actual behavior of both partners was determined by the same algorithm that modeled trustworthy behavior. When the first trust game was finished, the a priori information for the second trust game was shown and the second trust game was played. All participants played both trust games. Participants were debriefed via e-mail about the research aims and the key results.

\section{Behavioral data analysis}

\section{Manipulation checks}

Two tests were done to verify that the a priori information had the intended effects. First, a repeated-measures ANOVA with a Helmert contrast was carried out to test whether the investment made on the first trial in the consistent condition was significantly higher than the investments made during the acquaintance rounds. Second, for the inconsistent condition we tested whether the investment on the first trial was significantly lower than the investments made during the acquaintance rounds.

\section{Behavioral analysis}


The effect of gender and age group on baseline trust was examined using an independent t-test. The effect of social network position on baseline trust was tested using a linear regression analysis. Baseline trust is the investment made during the first trial. Differences in the perception on the trustworthiness of both partners was tested with a dependent t-test. Age group differences in the perception were tested using an independent t-test.

To answer the research aims, behavioral data analysis was performed in $\mathrm{R}$ version 3.5.1 using a mixed models approach. Mixed effects models take into account the repeated structure of the data, which allows for modeling the development of trust behavior over time. Mixed effects models comprise fixed and random effects. Fixed effects can be used to model mean development over time, while individual differences can be accounted for by random effects. A fixed intercept would capture the mean starting point, while a random intercept accounts for individual differences in starting points. Similarly, a fixed slope models the mean trajectory while a random slope accounts for individual differences in the trajectory. With regards to the current study, participants may differ in the starting points of the investments (captured by a random intercept) or in the change of investments over time (captured by a random slope).

Two model-fitting procedures were implemented. The first fitting procedure was performed to answer the first research aim examining how adolescents adapt their trust behavior and to answer the second aim whether the adaptation processes are different for young and late adolescents (the study sample is both the young and the late adolescent group). The second fitting procedure was performed to answer the third research aim whether the ability to adapt one's trust behavior is related to social network position (the study sample is the late adolescent group). Fixed effects or random effects were added in each step of the two model building procedures that are explained below. Only when the model fit improved as a result of adding fixed or random effects, 
the fixed or random effects were kept in the consecutive model. In the model building procedure, we controlled for the variable gender but the variable was removed when the model fit did not improve.

The first model fitting procedure was set up to answer the first and second research aims. The fitting procedure included the following steps. First, a null model consisting of a random intercept for the levels time, condition and participants was fitted. The predictor time was centralized. In model 1, both the linear and quadratic predictor of time were included as fixed effects. If model 1 showed a significant better fit to the data compared to the null model, we proceeded to fitting model 2 (see below for the criteria that were used to assess model fit). If the quadratic predictor of time was insignificant, it was removed in the subsequent model. In model 2, an interaction between time and condition was added as fixed effect. A random slope for time was included in model 3. In the final model, model 4 , the predictor age group was added as a fixed effect in a three-way interaction with time and condition. Age group was added as a binary variable. A second fitting procedure was set up to answer the third research aim. The null model and the first three models of this second model building approach are similar to the null model and the first three models in the first building approach described above. This means the null model consists of a random intercept for time, condition and participants. In model 1 the fixed effects of the linear and the quadratic predictor of time were added. An interaction between time and condition was added as fixed effect in model 2 and in model 3 a random slope for time was added. Then, in model 4 eigencentrality was added as a fixed effect in a three-way interaction with time and condition. The predictor eigencentrality was standardized into z-scores.

The experimental design was a crossed design. The R package lme4 and the supplementary package lmerTest were used for analysis as these packages are recommended for crossed designs 
(Bates et al., 2015; Kuznetsova et al., 2017). All models were fitted using a full maximum likelihood estimation method. Two criteria were used to assess model fit. First, a likelihood ratio test was used to compare a model with the previous model. A p-value below 0.05 indicated the model explained significantly more variance compared to the previous model. Second, Akaike Information Criterion (AIC) values were reviewed. Models with lower AIC were preferred. If the likelihood test and the AIC value favored a specific model compared to the previous model, the model fitting procedure was continued. For completeness, Bayesian Information Criterion (BIC) values are also provided.

\section{fMRI data acquisition}

MRI scans were acquired using a standard Philips 3T Achieva MRI scanner at the Spinoza Centre for Neuroimaging in Amsterdam. For each participant, fMRI data was collected during two experimental runs of T2*-weighted EPI. The runs consisted of a minimum of 317 and a maximum of 350 scans depending on the task duration (repetition time $(\mathrm{TR})=2 \mathrm{sec}$, echo time $(\mathrm{TE})=54 \mathrm{msec}$, ascending sequential acquisition, 37 slices, slice thickness $=3 \mathrm{~mm}$, gap thickness $=0.3 \mathrm{~mm}$, field of view $(\mathrm{FOV})=240 \times 240 \times 122 \mathrm{~mm}$, voxel size=3 $33 \times 3 \mathrm{~mm})$. A T1-weighted 3D multishot TFE anatomical scan was acquired $(\mathrm{TR}=8 \mathrm{msec}, \mathrm{TE}=3.9 \mathrm{msec}, 220$ slices, slice thickness $=1 \mathrm{~mm}$, $\mathrm{FOV}=240 \times 188 \times 220 \mathrm{~mm}$, voxel size=1 $=1 \times 1 \mathrm{~mm})$.

fMRI data analysis

fMRI analyses were done using Statistical Parametric Mapping 12 (https://www.fil.ion.ucl.ac.uk/spm/software/). The following preprocessing steps were performed. First, all functional images were realigned using a rigid body transformation with a least squared 
difference method between consecutive images. Second, the structural image was co-registered to an individualized mean image. Then the structural image was segmented and normalization parameters were estimated using unified segmentation. Next, these parameters were used to transform the functional images and the structural image into Montreal Neurological Institute (MNI) space. Last, smoothing was done using an $8 \mathrm{~mm}$ Gaussian kernel full width at half maximum.

A general linear model (GLM) was set up for every individual. The GLM consisted of two runs, one for each condition, and both included four regressors of interest, a regressor modeling the task periods of no interest and the six motion parameters. The investment regressor was modeled as the period from the start of the experimental trials up until the time point an investment was made (4-8 seconds, depending on the reaction time, see Figure 1). The control-selection regressor covered the period from start of the experimental trials up until a selection was made (48 seconds, depending on the reaction time, see Figure 1). The feedback regressor was defined as the time that the participant viewed the return of the partner and the outcome of the experimental trials (6-8 seconds, see in Figure 1). The control-feedback regressor consisted of the similar feedback period in the control trials (6-8 seconds, see Figure 1). The regressor of no interest was the time covering the period that the a priori information before the start of the experiment was presented (4 seconds) and the period covering the investment shown ( 2 seconds, see Figure 1$)$. The waiting screen and the fixation cross were not modeled (see Figure 1).

Next, for each subject an investment contrast image and a feedback contrast image for both conditions were created. The investment contrast was defined by comparing the investment phase of the experimental trials with the selection phase of the control trials and the feedback contrast was created comparing the feedback phase of the experimental trials with this similar phase of the 
control trials. To compare the two conditions, an additional investment and an additional feedback contrast were created by contrasting these phases between the consistent and inconsistent condition.

Following Lemmers-Jansen, Krabbendam, Veltman, and Fett (2017) a region of interest (ROI) analysis was done on nine well-described regions that have been previously associated with trust game paradigms. These regions with corresponding MNI coordinates are: the right TPJ (45, $-43,32)$, the left TPJ $(-44,-46,29)$, the right $\operatorname{dIPFC}(51,18,30)$, the right insula $(36,24,0)$, the $\operatorname{ACC}(-3,27,33)$, the right ventral striatum $(14,12,-5)$, the amPFC $(0,42,6)$, the right caudate $(6$, $11,5)$ and the left caudate $(-7,12,-4)$. ROIs were created by $5 \mathrm{~mm}$ spheres centered around the coordinates of the ventral striatum and caudate, and $10 \mathrm{~mm}$ spheres around the remaining coordinates. Using the MarsBar version 0.44 toolbox (http://marsbar.sourceforge.net) contrast values within these ROIs were extracted and tested. Additionally, an exploratory whole-brain analysis was done for all contrasts.

The contrasts were tested using the Simple Interactive Statistical Analysis Bonferroni tool (SISA, http://www.quantitativeskills.com/sisa/calculations/bonfer.htm) to control for multiple testing for the ROIs. The standard Bonferroni correction is too stringent as it does not take into account the correlations between contrast values. The SISA tool takes into account the correlation between the ROIs separately for each contrast and this results in a different alpha level for each contrast. The four within-condition contrasts and the two between-condition contrasts were used to answer the first research aim on the neural mechanisms of trust. For the second research aim, we expected the late adolescents to show increased activation in regions of interest during the inconsistent condition compared to the young adolescents. Therefore, the contrast values of the ROIs of the investment and of the feedback contrast of the inconsistent condition were regressed 
on the between factor age and the within factor ROI in a factorial mixed ANOVA. The SISA tool was used to control for multiple testing using post-hoc t-tests. Concerning the third research aim, we expected adolescents with more central positions to show faster adaptation behavior towards the inconsistent partner and that this will be accompanied by increased brain activity in areas related to mentalizing. To this end, the contrast values of the amPFC, left TPJ and right TPJ of the investment contrast of the inconsistent condition were regressed on the eigencentrality values using a regression analysis. We only looked at the investment contrast and the amPFC, left TPJ and right TPJ as we have hypotheses about this contrast and about these ROIs. If case there is an effect of age and eigencentrality, we repeated the analyses in the consistent condition to examine if the effect was specific to the inconsistent condition.

\section{Results}

\section{Behavioral results}

\section{Manipulation checks}

A Helmert contrast in a repeated-measures ANOVA showed that the investment during the first trial of the consistent condition was significantly higher compared to the investments during the three acquaintance rounds $(F(1,49)=11.83, p=0.001)$. The investment during the first trial of the inconsistent condition was significantly lower than the investments during the three acquaintance rounds $(F(1,49)=9.45, p=0.003)$. It was therefore concluded that the manipulation to create the impression of a consistent partner and an inconsistent partner was successful.

\section{Behavioral analysis}


Mean investments and standard deviation per trust game trial can be found in the supplementary materials. Multi-level analysis showed the best model fit for model 4 which included the three-way interaction between time, condition and age group. Results of the full procedure are shown in Table $2 \mathrm{a}$ and a full description of model 4 is shown in Table $2 \mathrm{~b}$. Regarding the first research aim in which we examined how adolescents adapt their trust behavior, model 4 showed that the interaction between time and condition was significant indicating that the effect of time is different in the two conditions. Follow-up analyses indicate no significant effect of time in the consistent condition $(t(29)=-1.08, p=0.29)$ and a quadratic effect of time in the inconsistent condition $(t(900)=-2.6, p<0.01)$. Concerning the second research aim, namely whether these behavioral adaptation processes are different for young and late adolescents, the three-way interaction between time, condition and age group in model 4 was significant indicating that the effect of time differed between the conditions and that the effect of time within the conditions also differed per age group, see Figure 2. To further interpret the three-way interaction, a follow-up analysis investigating the interaction between time and age group was done for both conditions separately. Concerning the inconsistent condition, there was a trend towards an interaction between the linear effect of time and age group, showing that late adolescents have a higher increase of investments over time compared to the young adolescents, $(t(50)=-1.83, p=0.07)$. For the consistent condition there was a trend towards an interaction between time and age group that indicated that the young adolescents showed a steeper decrease of investments over time compared to the late adolescents, $(t(50)=-1.98, p=0.05)$. In both conditions there were no gender and age differences in baseline trust (gender consistent condition: $p=0.74$, gender inconsistent condition: $p=0.94$, age consistent condition: $p=0.10$, age inconsistent condition: $p=0.69$ ). The consistent partner was rated as significantly more trustworthy compared to the inconsistent partner 
$(t(47)=2.07, p=0.04)$. The young adolescents and the late adolescents did not differ in their perception of the trustworthiness of the partners (consistent condition: $p=0.46$, inconsistent condition: $p=0.39)$.

A second model building procedure was performed to examine whether the change in trust behavior is related to eigencentrality in late adolescents (research aim 3). The best fitting model was model 4. Results of the full procedure are shown in Table $2 \mathrm{a}$ and a full description of the model in Table 2c. Results of model 4 showed that the interaction between the quadratic effect of time, condition and eigencentrality was significant, see Figure 3. The three-way interaction indicated that the effect of time is different in both conditions and that this effect is different for people with differing eigencentrality scores. A follow-up analysis was done for both conditions separately to further interpret the three-way interaction. For the inconsistent condition there was a significant interaction between the linear effect of time and eigencentrality showing a negative association between eigencentrality and time $(t(29))=-3, p<0.01)$. This means that participants with a lower eigencentrality showed a steeper increase of investments over time compared to participants with a higher eigencentrality. There was no effect of eigencentrality ( $p=0.99)$ or interaction effect between eigencentrality and time $(p=0.96)$ in the consistent condition. Furthermore, there was no effect of eigencentrality on baseline trust in the consistent condition $(p=0.73)$ while a positive effect of eigencentrality was borderline significant on baseline trust in the inconsistent condition $(t(1,24)=2.09, p=0.05)$. 
Trust behavior and friendships

Table 2a

Results model building procedure age

\begin{tabular}{lccccc}
\hline & Null model & Model 1 & Model 2 & Model 3 & Model 4 \\
\hline & AIC BIC & AIC BIC & AIC BIC & AIC BIC & AIC BIC \\
\hline $\begin{array}{l}\text { Model building procedure } \\
\text { (Age) }\end{array}$ & 87158743 & 87128756 & 86888744 & 86698736 & 86678767 \\
$\begin{array}{l}\text { Model building procedure } \\
\text { (Eigencentrality) }\end{array}$ & 44834508 & 44734513 & 44554504 & 44344494 & 44244513 \\
\hline
\end{tabular}


Trust behavior and friendships

Table $2 b$

Results fit model 4

Variance

0.000

Intercept condition

0.000

Intercept subjects

2.178

Slope time

0.003

Fixed effects

Intercept

$6.767 * *$

0.987

1.651

$-0.629 * *$

$-1.01 *$

Age

Cross-level interactions

Time linear*Condition

Time quadratic*Condition

Time linear*Age

Time quadratic*Age

Condition*Age

Time linear*Condition*Age

Time quadratic*Condition*Age
$18.972 * * \quad 3.86(<0.01) \quad 9.334 \quad 28.61$

$\begin{array}{llll}-11.584 * & 0.760(0.019) & -21.222 & -1.946\end{array}$

$\begin{array}{llll}-14.639 * & -2.023(0.045) & -28.947 & -0.331\end{array}$

$\begin{array}{llll}-8.661 & -1.453(0.146) & -20.349 & 3.026\end{array}$

$\begin{array}{llll}0.049 & 0.262(0.793) & -0.32 & 0.419\end{array}$

$\begin{array}{llll}-1.853 & -0.220(0.826) & -18.381 & 14.676\end{array}$

$16.791 * \quad 1.992(0.046) \quad 0.262 \quad 33.32$

*two-tailed, $\mathrm{p}<0.05 . * *$ two-tailed, $\mathrm{p}<0.01$. 
Trust behavior and friendships

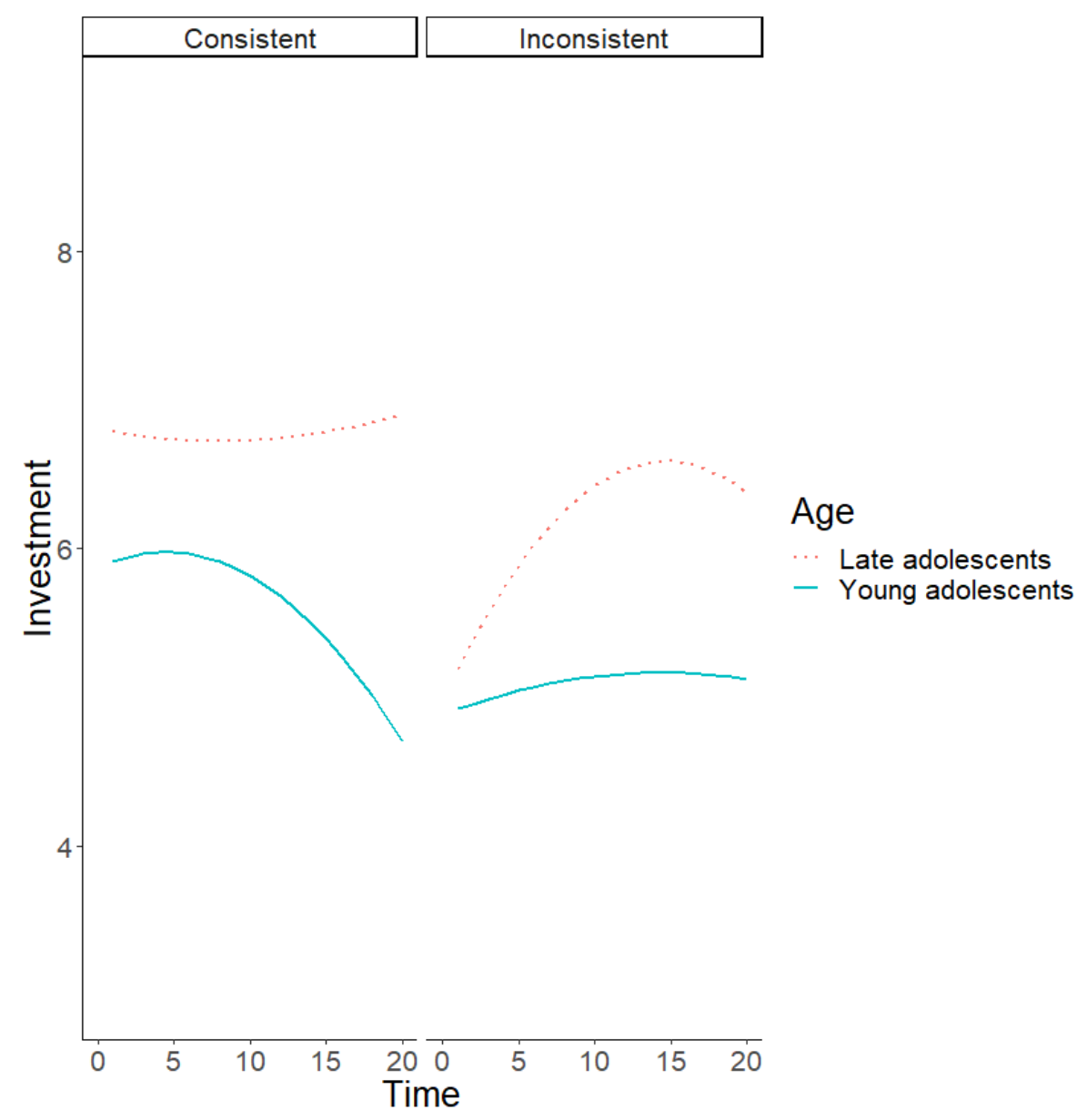

Figure 2. Significant three-way interaction between time (i.e. trials during the trust game), condition and age. 
Trust behavior and friendships

Table 2c

Results fit model 4

Variance

0.000

Intercept condition

0.000

Intercept subjects

3.102

Slope time

0.005

\begin{tabular}{ll}
\hline Random effects & \\
Intercept time & 0.000 \\
Intercept condition & 0.000 \\
Intercept subjects & 3.102 \\
Slope time & 0.005
\end{tabular}

Fixed effects

Intercept

$7.054 *$

1.506

3.270

$-0.7 *$

$-0.003$

Eigencentrality

Cross-level interactions

Time linear*Condition

Time quadratic*Condition

Time linear*Eigencentrality

Time quadratic*Eigencentrality

Condition*Eigencentrality

Time linear*Condition*

Eigencentrality

Time quadratic*Condition* Eigencentrality
$\mathrm{T}$ value (p- 95\% CI

value)

\section{Upper}


Trust behavior and friendships

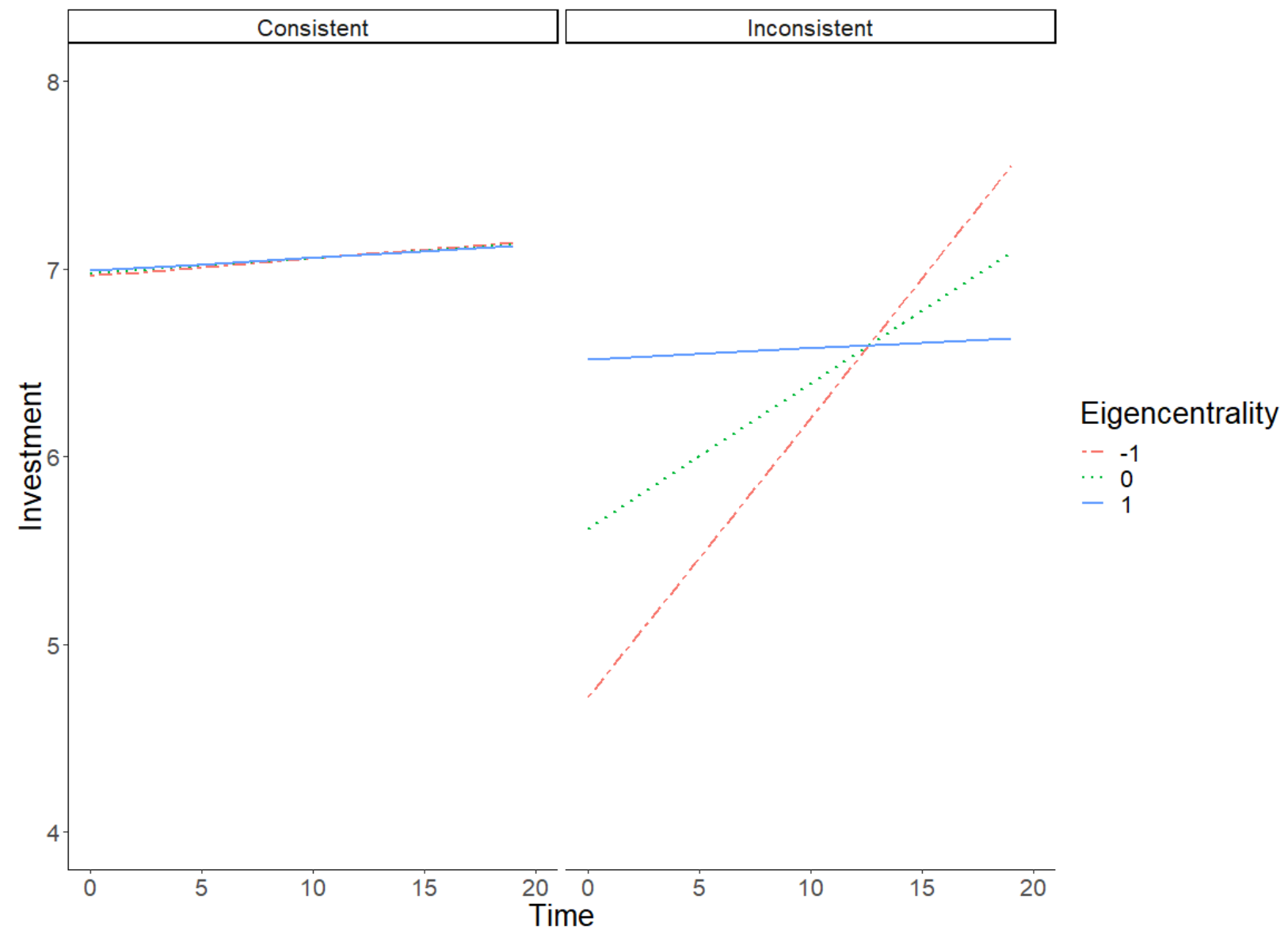

Figure 3. Significant three-way interaction between time (i.e. trials during the trust game), condition and eigencentrality. For visual purposes, eigencentrality is displayed using z-scores. 
Trust behavior and friendships

fMRI analyses

ROI analysis

The first research aim was to examine the neural mechanisms of trust during the trust game. The following ROIs showed significant activation during the investment phase of the consistent condition relative to the control trials: the left ventral striatum, the left amPFC, the right ACC and the left and right caudate. The same ROI results were found for the investment phase of the inconsistent condition relative to the control trials. There was no significant between-condition effect. See Table 4 for the results of these ROI analyses.

Regarding the feedback phase, the left and right TPJ, the right dIPFC, the right ACC, the right insula, the right caudate and the left ventral striatum showed increased activity during the inconsistent condition relative to the control trials. The same ROI results, except for the left ventral striatum, were found for the consistent condition relative to the control trials. There was a significant between-condition effect for the left TPJ and right dIPFC showing more activation during the feedback phase of the inconsistent condition compared to consistent condition, see Figure 4. See Table 5 for the results of these ROI analyses.

Additional whole-brain analyses of the investment phase of both conditions showed increased activity in the medial prefrontal cortex, precuneus, bilateral parietal regions, bilateral visual regions, bilateral motor regions and bilateral striatum. No significant results were found when the investment phase of the inconsistent condition was contrasted with the investment phase of the consistent condition. For the feedback phase of both conditions, the whole-brain analysis showed increased activity in the dorsolateral and ventrolateral prefrontal cortex, dorsomedial prefrontal cortex, bilateral insula, bilateral striatum, bilateral parietal regions, bilateral visual 
regions and bilateral motor regions. No significant results were found when the feedback phase of the inconsistent condition was contrasted with the investment phase of the consistent condition. Figures displaying the brain activity changes concerning the whole-brain analyses can be found in the supplementary materials.

Table 4

$P$-values for the three contrasts of the ROI analysis for the investment phase.

\begin{tabular}{llll}
\hline ROI (MNI coordinates) & $\begin{array}{l}\text { Inconsistent } \\
\text { control } \\
\text { investment phase }\end{array}$ & $\begin{array}{l}\text { vs. } \\
\text { investment phase }\end{array}$ & $\begin{array}{l}\text { Consistent vs. control } \\
\text { inconsistent } \\
\text { investment phase }\end{array}$ \\
\hline Right TPJ $(45,-43,32)$ & 0.053765 & $0.020226^{* *}$ & 0.096338 \\
Left TPJ $(-44,-46,29)$ & 0.687735 & 0.250663 & 0.133586 \\
Right dIPFC $(51,18,30)$ & 0.357637 & 0.365009 & 0.196657 \\
Right insula $(36,24,0)$ & 0.482161 & 0.144141 & 0.272219 \\
ACC $(-3,27,33)$ & $0.0000000^{*}$ & $0.000000^{* *}$ & 0.553735 \\
Right ventral striatum $(14,12,-5)$ & $0.0000000^{*}$ & $0.000000^{* *}$ & 0.397716 \\
amPFC $(0,42,6)$ & $0.000126^{*}$ & $0.000000^{* *}$ & 0.543467 \\
Right caudate $(6,11,5)$ & $0.000028^{*}$ & $0.001232^{* *}$ & 0.288658 \\
Left caudate $(-7,12,-4)$ & $0.000078^{*}$ & $0.000001^{* *}$ & 0.460933 \\
\hline
\end{tabular}

*significant at adjusted alpha level $=0.0363584$. $* *$ significant at adjusted alpha level $=0.0373298$. 

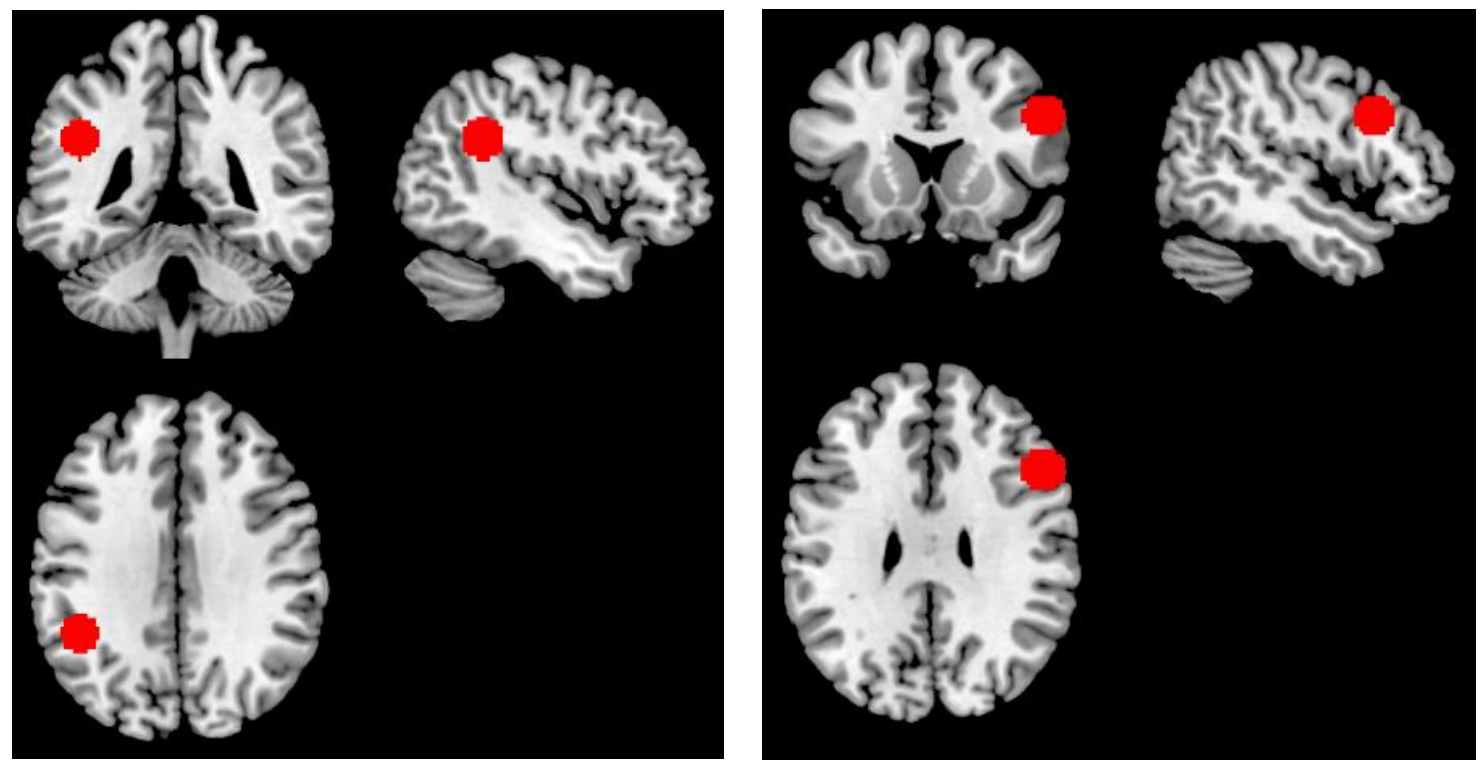

Figure 4. The left TPJ (left, $M=0.06, S D=0.22$ ) and the right dlPFC (right, $M=0.11, S D=0.39$ ) are the two regions of interest that show increased activity during the feedback phase of the inconsistent condition compared to consistent condition. 
Trust behavior and friendships

Table 5

P-values for the three contrasts of the ROI analysis for the feedback phase.

\begin{tabular}{llll}
\hline ROI (MNI coordinates) & $\begin{array}{l}\text { Inconsistent } \\
\text { control } \\
\text { feedback phase }\end{array}$ & $\begin{array}{l}\text { vs. } \\
\text { Consistent vs. control } \\
\text { feedback phase }\end{array}$ & $\begin{array}{l}\text { Inconsistent } \\
\text { consistent } \\
\text { feedback phase }\end{array}$ \\
\hline Right TPJ $(45,-43,32)$ & $0.000000^{*}$ & $0.000000^{* *}$ & 0.060202 \\
Left TPJ $(-44,-46,29)$ & $0.000301^{*}$ & $0.000005^{* *}$ & $0.023568^{* * *}$ \\
Right dlPFC $(51,18,30)$ & $0.000000^{*}$ & $0.000000^{* *}$ & $0.030647 * *$ \\
Right insula $(36,24,0)$ & $0.000000^{*}$ & $0.000000^{* *}$ & 0.066841 \\
ACC $(-3,27,33)$ & $0.000002^{*}$ & $0.000000^{* *}$ & 0.421323 \\
Right ventral striatum (14, 12, -5) & $0.000027^{*}$ & $0.015017 * *$ & 0.222222 \\
amPFC $(0,42,6)$ & 0.625867 & 0.515449 & 0.311450 \\
Right caudate $(6,11,5)$ & $0.000001^{*}$ & $0.000002^{* *}$ & 0.399594 \\
Left caudate $(-7,12,-4)$ & 0.566911 & 0.518378 & 0.455787
\end{tabular}

$*$ significant at adjusted alpha level=0.0328632. **significant at adjusted alpha level=0.0320784.

$* * *$ significant at adjusted alpha level $=0.0354902$.

Age effects

An interaction between ROI and age was performed using a mixed factorial ANOVA to test the second aim of whether there are age differences in the neural mechanisms underlying trust during interactions in which the preprogrammed partner behaves differently than expected. No age effects were found for the investment phase of the inconsistent condition relative to the control trials (inconsistent condition: $p=0.73$ ). For the feedback phase of the inconsistent condition relative to the control trials, a significant interaction between ROI and age was found $(F(8,48)=3, p<0.05)$. Post-hoc t-tests revealed a significant effect of age on the contrast values of the dlPFC indicating 
the late adolescents showed more dlPFC activity than the young adolescents $(t(48)=-2.46, p<0.05)$. There was no significant age effect in the feedback phase of the consistent condition relative to the control trials $(p=0.5)$.

\section{Position in a social network}

For the third research aim we examined for the late adolescent group whether social network positions affect trust behavior and the neural mechanisms of trust during interactions with a preprogrammed partner that behaves differently than expected. Eigencentrality was not significantly associated with activity in the amPFC, left TPJ or right TPJ during the investment phase of the inconsistent condition $(p=0.827)$.

\section{Discussion}

In the current study we used a trust game to investigate adolescent's ability to adapt their trust behavior and the neural mechanisms of trust, developmental differences in trust behavior and in the neural mechanisms, and the relationship between trust behavior, neural mechanisms of trust and the position in a social network. The behavioral results showed that adolescents adapted their trust behavior during the course of the game, that there were age-related differences in these adaptation processes, that late adolescents with less central network positions were more adaptive in their trust behavior compared to adolescents with more central network positions and a trend result that network positions and baseline trust are positively related. Neuroimaging analyses showed that the left TPJ and right dlPFC were more active during feedback processing in the inconsistent condition compared to the consistent condition. In addition, we found increased activity in the dlPFC in the late compared to the young adolescent group, when trusting a partner 
that behaves more trustworthily than was expected. No associations were found between neural activity and measures of social network position.

\section{Adaptation of trust behavior and neural mechanisms of trust}

The current study showed that baseline trust behavior can be manipulated by providing information about the partner prior to the interaction. In answering the first research aim of the study, it was shown that adolescents adapt their trust behavior adequately in response to the behavior of the partner. Specifically, when the interaction partner behaved as the a priori information suggested, there was no change in trust behavior over time. When the interaction partner was more trustworthy than a priori information suggested, the adolescents increased their trust over the course of the game, with trust behavior stabilizing towards the end of the interaction. This demonstrates that adolescents are able to flexibly adapt their behavior in social situations. Interestingly, reports on post-hoc questionnaires indicated that the partner that behaved as expected was perceived as significantly more trustworthy compared to the partner that behaved differently than expected.

Furthermore, in answering the first research aim of the study in which we examine neural mechanisms of trust, it was shown that results are in accordance with previous studies and suggest that regions associated with mentalizing, cognitive control and reward processing are implicated in the trust game (Bellucci et al., 2017; Delgado et al., 2005; Fett et al., 2012; King-Casas et al., 2005). In both conditions trust decisions were associated with increased activity in the left amPFC, the right ACC, the left ventral striatum and the left and right caudate. When making a trust decision the trustor considers how much to invest by taking into account the partner's trustworthiness and intentions, thus recruiting mentalizing processes. Activity in the left and right TPJ, the right dlPFC, 
the right $\mathrm{ACC}$, the right insula and the right caudate increased in both conditions during the phase the partner's return was received. Additionally, activity in the ventral striatum was increased in the inconsistent condition only. During the time the feedback was received, conflict monitoring and reward processes may have been recruited as the trustor processes the partner's return in relation to their own investments. Based on these ROI results, it seems that mentalizing processes are involved in the investment phase of the trust game as well as in the feedback phase as activity in mentalizing-related areas, such as the TPJ, was increased in both phases. Similarly, reward processes and conflict monitoring processes are involved in both phases of this multi-round version of the game as in both phases activity in reward-related and conflict monitoring related areas, such as the caudate, insula, dlPFC and ACC, were increased.

In line with our hypotheses, increased activation in the inconsistent condition compared to the consistent condition was observed in the left TPJ and the dlPFC during the feedback phase of the trust game. The TPJ is an area that in the context of the trust game and other social decisionmaking paradigms, has been linked to mentalizing behavior, specifically redirecting the attention from oneself to someone else (Fett et al., 2012; Lamm et al., 2007; Van Den Bos et al., 2009). This may tentatively suggest that more mentalizing processes were elicited in the condition in which a priori information was discrepant from actual behavior. We further observed increased activity in the dlPFC during the feedback phase of the inconsistent condition compared to the consistent condition, which has been related to cognitive control processes and decision making (MacDonald et al., 2000; Ridderinkhof et al., 2004). No significant differences were found when the investment phase of the two conditions were compared. This means the hypothesis that trust decisions in the inconsistent condition would be associated with increased activity in mentalizing-related areas compared to trust decisions in the consistent condition was not confirmed. 
Adaptation of trust behavior and the neural mechanisms of trust: developmental differences

Regarding the second research aim on developmental differences in the adaptation of trust, it was shown that the way in which young and late adolescents adapted their trust behavior differed significantly. In line with our hypotheses, post-hoc analyses showed a trend suggesting that when interacting with a partner that is more trustworthy than a priori information suggested, late adolescents increased their trust behavior faster throughout the game than young adolescents. This tentatively suggests that late adolescents are more skilled in adapting their behavior in response to others than young adolescents. This could be explained by the better developed mentalizing abilities of late adolescents, which would allow them to better infer the motives of a partner and to understand behavior that is divergent from what was expected and consequently adapt their own behavior. Also, post-hoc analyses showed a trend result that throughout the game young adolescents, compared to late adolescents, decreased their trust behavior more towards a partner that behaved in line with the a priori information. This finding was not hypothesized, but may tentatively suggest a general developmental effect that young adolescents showed less adequate responses to the actual behavior of a partner compared to late adolescents. This was not caused by the young adolescents finding the partner less trustworthy, as shown by a lack of significant differences in the trustworthiness rating between the two groups. In support of our findings, results of previous studies also reported more adequate trust behavior over repeated interactions with increasing age in adolescence (Fett et al., 2012; van den Bos et al., 2010).

Furthermore, in answering the second research aim on developmental differences in the neural mechanisms of trust during interactions in which a preprogrammed partner behaves differently than expected, no effect of age was found during the investment phase in the 
inconsistent condition. In line with our hypotheses, age effects were found during the feedback phase of the inconsistent condition, as shown by increased dIPFC activity in the late adolescents compared to the young adolescents. These findings are supported by an earlier trust game study in which age-related increases in the dIPFC were found in participants from the ages 16 to 27 (Lemmers-Jansen et al., 2017). The dIPFC has been related to cognitive control and conflict monitoring processes in social dilemmas (Declerck et al., 2013; Greene et al., 2004; MacDonald et al., 2000; Ridderinkhof et al., 2004). In the current study, the partner in the inconsistent condition showed behavior that was not in line with the a priori information, thereby creating uncertainty and conflict between expected and observed behavior. Perhaps the late adolescents had a better understanding of the behavior and the intentions of the partner both before the start of the game and during the game. This may have resulted in more uncertainty and conflict processes, hence increased dlPFC activation. In line with this suggestion, the behavioral findings showed a trend result of a stronger increase of trust behavior throughout the game for the late adolescents compared to the young adolescents. No age effects were found in the consistent condition.

\section{Trust and social network positions}

Regarding the third research aim, how social network positions of late adolescents relate to behavioral adaptation processes and neural mechanisms of trust during interactions in which a preprogrammed partner behaves differently than expected, we found some support for a relationship between the position in a social network of friendships in daily life and trust behavior. Contrary to expectations, less central adolescents increased their investments over time more so than adolescents with more central positions, indicating that less central adolescents adapted their trust behavior over the course of the game more so. A post-hoc explanation for this unexpected 
finding may lie in the different degrees of power associated with low and high levels of centrality in network positions. That is, social relations between people imply a certain degree of power and dependency between the actors involved (Emerson, 1962). For example, highly central people are said to be influential people because of their connections to well-connected others. People with fewer connections are dependent on central people to realize goals and fulfill needs, and, they may therefore be more motivated to accurately infer other people's perspective. Experimental research has shown that being able to influence others and that feelings of having power over others is related to a decrease in perspective taking, mentalizing and concern for others (Galinsky et al., 2006; Galinsky et al., 2016). Therefore, adolescents with central positions may have had less concern for and paid less attention to the a priori information and to the following trustworthy behavior. Conversely, the adolescents with less central positions may be less influential in the friendship network and therefore be more observant in noticing an inconsistency between the a priori information describing the untrustworthy behavior and the partner's actual trustworthy behavior. Compared to adolescents with central positions, adolescents with a less central positions may not necessarily be better skilled at taking someone else's perspective, but they may have a higher propensity for perspective taking as they are more dependent and focused on other people. Finally, we did not observe any associations between network positions and activation in the regions of interest. A larger sample may be required to relate real-life measures such as friendships networks to brain mechanisms. Also, perhaps to identify the neural mechanisms associated with network positions, one may need to include participants who have either a very central position or a very peripheral position.

\section{Limitations and future directions}


The present study has some limitations. First, the sample size was relatively small which may have precluded the detection of individual differences related to age or social network. A suggestion is to use a longitudinal design to investigate directional influences whether having a certain position in the network changes your behavior or whether the position in a network follows certain behavior. A second limitation is the deception used in the trust game. This was necessary for the experimental design and all participants were debriefed after the study. Third, trial-by-trial feedback was not examined in the current analysis as the learning curve of trust behavior may be different for participants. Nevertheless, the interest in the current study was specifically to study the effect of context (a priori information) on trust behavior and not trial-by-trial learning.

\section{Conclusions}

The current study shows that adolescents adapt their trust behavior over the course of a social interaction and that these adaptation processes differ between late adolescents and young adolescents. The results tentatively suggest that late adolescents can more flexibly adapt their behavior towards a partner by overcoming information that falsely described that partner and this was supported by increased dIPFC activity related to conflict-monitoring processes. Also, the current study adds to the literature by showing that adolescents with different social network positions respond differently to social interactions in which their expectation about the partner does not match the actual behavior of the partner. Future research may build on this finding to deepen the understanding about the relationship between trust behavior and different positions in a social network.

\section{Acknowledgments}


The study was supported by an European Research Council Consolidator Grant to L. K. [Grant number 648082], an European Research Council Starting Grant to N. v. A. [Grant number 71673], and a Marie Curie Individual Fellowship of the EU Horizon2020 Framework Program to M.R.T. v. K. [Grant \#704506]. We would like to thank Miriam Hollarek for her assistance in data collection, and all the adolescents, parents and schools for their participation. 


\section{References}

Bailey, P. E., Szczap, P., McLennan, S. N., Slessor, G., Ruffman, T., \& Rendell, P. G. (2016). Age-related similarities and differences in first impressions of trustworthiness. Cognition and Emotion, 30(5), 1017-1026.

Bates, D., Maechler, M., Bolker, B., \& Walker, S. (2015). Fitting linear mixed-effects model using Ime4. Journal of Statistical Software, 67, 1-48.

Bellucci, G., Chernyak, S. V., Goodyear, K., Eickhoff, S. B., \& Krueger, F. (2017). Neural signatures of trust in reciprocity: A coordinate-based meta-analysis. Human Brain Mapping, 38(3), 1233-1248.

Berg, J., Dickhaut, J., \& McCabe, K. (1995). Trust, reciprocity, and social history. Games and Economic Behavior, 10(1), 122-142.

Borgatti, S. P., Everett, M. G., \& Freeman, L. C. (2002). Ucinet for windows: Software for social network analysis. Harvard, MA: Analytic Technologies.

Burt, R. S., Kilduff, M., \& Tasselli, S. (2013). Social network analysis: Foundations and frontiers on advantage. Annual Review of Psychology, 64, 527-547.

Buskens, V. (1998). The social structure of trust. Social Networks, 20(3), 265-289.

Cillessen, A. H., \& Marks, P. E. (2011). Conceptualizing and measuring popularity. In Popularity in the peer system (pp. 25-56). Guilford Press.

Declerck, C. H., Boone, C., \& Emonds, G. (2013). When do people cooperate? The neuroeconomics of prosocial decision making. Brain and Cognition, 81(1), 95-117.

Delgado, M. R., Frank, R. H., \& Phelps, E. A. (2005). Perceptions of moral character modulate the neural systems of reward during the trust game. Nature Neuroscience, 8(11), 1611.

Emerson, R. M. (1962). Power-dependence relations. American Sociological Review, 31-41.

Fareri, D. S., Chang, L. J., \& Delgado, M. R. (2012). Effects of direct social experience on trust decisions and neural reward circuitry. Frontiers in Neuroscience, 6, 1-17.

Fett, A.-K. J., Gromann, P. M., Giampietro, V., Shergill, S. S., \& Krabbendam, L. (2012). Default distrust? An fMRI investigation of the neural development of trust and cooperation. Social Cognitive and Affective Neuroscience, 9(4), 395-402.

Fouragnan, E., Chierchia, G., Greiner, S., Neveu, R., Avesani, P., \& Coricelli, G. (2013). Reputational priors magnify striatal responses to violations of trust. Journal of Neuroscience, 33(8), 3602-3611.

Galinsky, A. D., Magee, J. C., Inesi, M. E., \& Gruenfeld, D. H. (2006). Power and perspectives not taken. Psychological Science, 17(12), 1068-1074. 
Trust behavior and friendships

Galinsky, A. D., Rucker, D. D., \& Magee, J. C. (2016). Power and perspective-taking: A critical examination. Journal of Experimental Social Psychology, 67, 91-92.

Greene, J. D., Nystrom, L. E., Engell, A. D., Darley, J. M., \& Cohen, J. D. (2004). The neural bases of cognitive conflict and control in moral judgment. Neuron, 44(2), 389-400.

King-Casas, B., Tomlin, D., Anen, C., Camerer, C. F., Quartz, S. R., \& Montague, P. R. (2005). Getting to know you: reputation and trust in a two-person economic exchange. Science, 308(5718), 78-83.

Kuznetsova, A., Brockhoff, P. B., \& Christensen, R. H. B. (2017). ImerTest Package: Tests in Linear Mixed Effects Models. Journal of Statistical Software, 82, 1-26.

Lamm, C., Batson, C. D., \& Decety, J. (2007). The neural substrate of human empathy: effects of perspective-taking and cognitive appraisal. Journal of Cognitive Neuroscience, 19(1), 42-58.

Lee, N. C., Jolles, J., \& Krabbendam, L. (2016). Social information influences trust behaviour in adolescents. Journal of Adolescence, 46, 66-75.

Lemmers-Jansen, I. L., Fett, A.-K. J., Shergill, S. S., Van Kesteren, M. T., \& Krabbendam, L. (2019). Girls-boys an investigation of gender differences in the behavioral and neural mechanisms of trust and reciprocity in adolescence. Frontiers in Human Neuroscience, 13, 780-790.

Lemmers-Jansen, I. L., Krabbendam, L., Veltman, D. J., \& Fett, A.-K. J. (2017). Boys vs. girls: Gender differences in the neural development of trust and reciprocity depend on social context. Developmental Cognitive Neuroscience, 25, 235-245.

MacDonald, A. W., Cohen, J. D., Stenger, V. A., \& Carter, C. S. (2000). Dissociating the role of the dorsolateral prefrontal and anterior cingulate cortex in cognitive control. Science, 288(5472), 1835-1838.

Marks, P. E., Babcock, B., Cillessen, A. H., \& Crick, N. R. (2013). The effects of participation rate on the internal reliability of peer nomination measures. Social Development, 22(3), 609-622.

Phan, K. L., Sripada, C. S., Angstadt, M., \& McCabe, K. (2010). Reputation for reciprocity engages the brain reward center. Proceedings of the National Academy of Sciences, 107(29), 13099-13104.

Ridderinkhof, K. R., Van Den Wildenberg, W. P., Segalowitz, S. J., \& Carter, C. S. (2004). Neurocognitive mechanisms of cognitive control: the role of prefrontal cortex in action selection, response inhibition, performance monitoring, and reward-based learning. Brain and Cognition, 56(2), 129140.

Robins, G. (2015). Doing social network research: Network-based research design for social scientists. Sage.

Sutter, M., \& Kocher, M. G. (2007). Trust and trustworthiness across different age groups. Games and Economic Behavior, 59(2), 364-382. 
van den Bos, W., Crone, E. A., Meuwese, R., \& Güroğlu, B. (2018). Social network cohesion in school classes promotes prosocial behavior. PloS One, 13(4), 1-16.

Van Den Bos, W., van Dijk, E., Westenberg, M., Rombouts, S. A., \& Crone, E. A. (2009). What motivates repayment? Neural correlates of reciprocity in the Trust Game. Social Cognitive and Affective Neuroscience, 4(3), 294-304.

van den Bos, W., van Dijk, E., Westenberg, M., Rombouts, S. A., \& Crone, E. A. (2011). Changing brains, changing perspectives: The neurocognitive development of reciprocity. Psychological Science, 22(1), 60-70.

van den Bos, W., Westenberg, M., van Dijk, E., \& Crone, E. A. (2010). Development of trust and reciprocity in adolescence. Cognitive Development, 25(1), 90-102.

Van't Wout, M., \& Sanfey, A. G. (2008). Friend or foe: The effect of implicit trustworthiness judgments in social decision-making. Cognition, 108(3), 796-803.

Volz, K. G., Schubotz, R. I., \& von Cramon, D. Y. (2005). Variants of uncertainty in decision-making and their neural correlates. Brain Research Bulletin, 67(5), 403-412.

Wardle, M. C., Fitzgerald, D. A., Angstadt, M., Sripada, C. S., McCabe, K., \& Phan, K. L. (2013). The caudate signals bad reputation during trust decisions. PloS One, 8(6), 1-9.

Zarolia, P., Weisbuch, M., \& McRae, K. (2017). Influence of indirect information on interpersonal trust despite direct information. Journal of Personality and Social Psychology, 112(1), 1-19. 

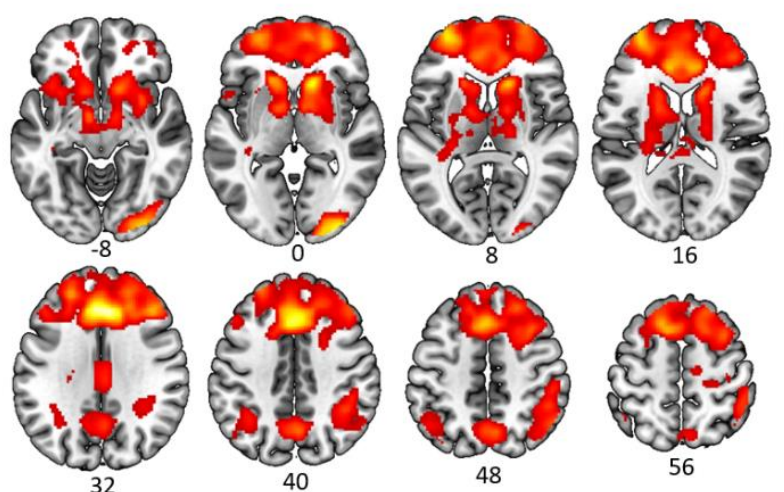

48
16
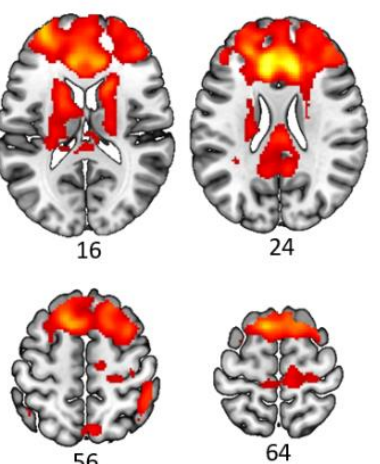

56

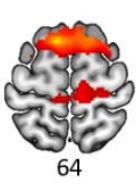

64
12

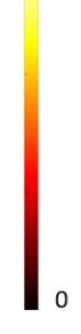

Figure for supplementary materials: Results whole-brain analysis consistent condition versus control condition during the investment phase (cluster defining threshold of $\mathrm{p}<0.001$, cluster probability of $\mathrm{p}<0.05$, family wise error corrected). Activity changes are overlaid on an anatomical MNI template. Color bars indicates t-values. The numbers below the brain slices indicate z-coordinates. 


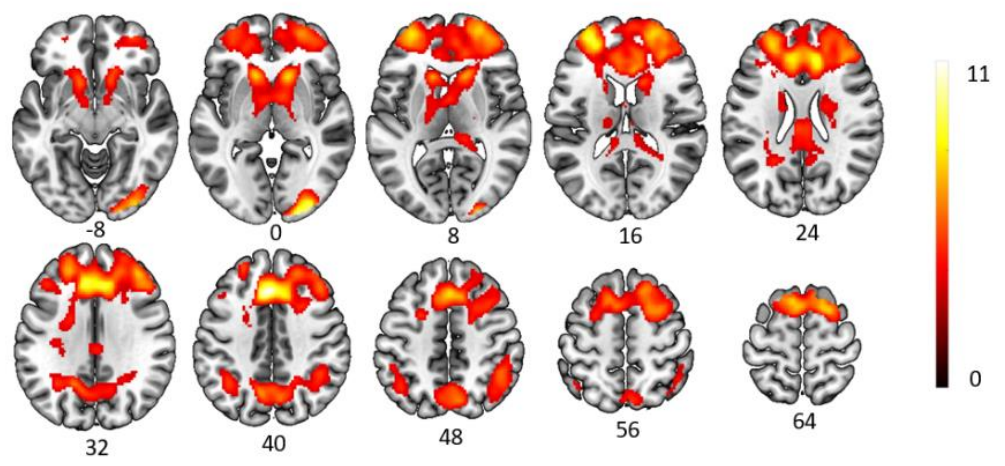

Figure for supplementary materials: Results whole-brain analysis inconsistent condition versus control condition during the investment phase (cluster defining threshold of $p<0.001$, cluster probability of $\mathrm{p}<0.05$, family wise error corrected). Activity changes are overlaid on an anatomical MNI template. Color bars indicates t-values. The numbers below the brain slices indicate z-coordinates. 


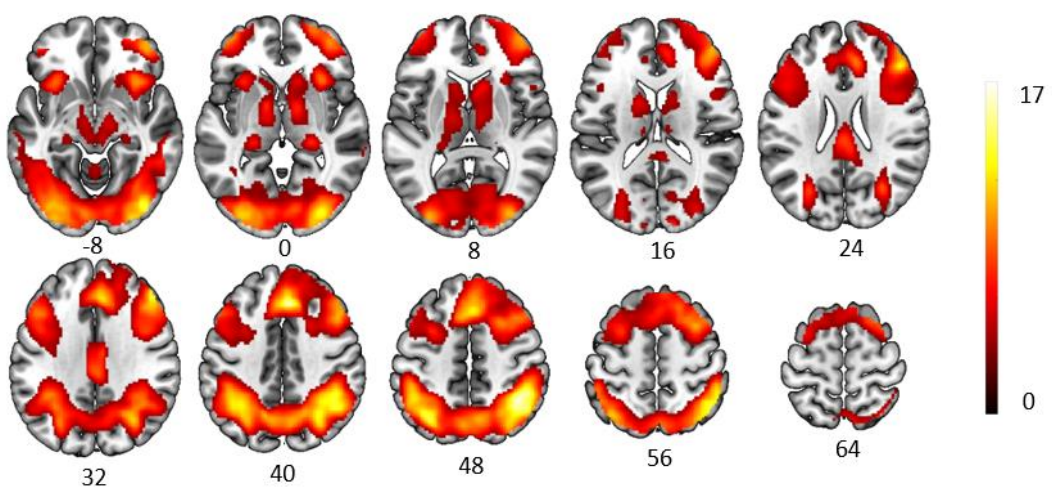

Figure for supplementary materials: Results whole-brain analysis consistent condition versus control condition during the feedback phase (cluster defining threshold of $\mathrm{p}<0.001$, cluster probability of $\mathrm{p}<0.05$, family wise error corrected). Activity changes are overlaid on an anatomical MNI template. Color bars indicates t-values. The numbers below the brain slices indicate z-coordinates. 


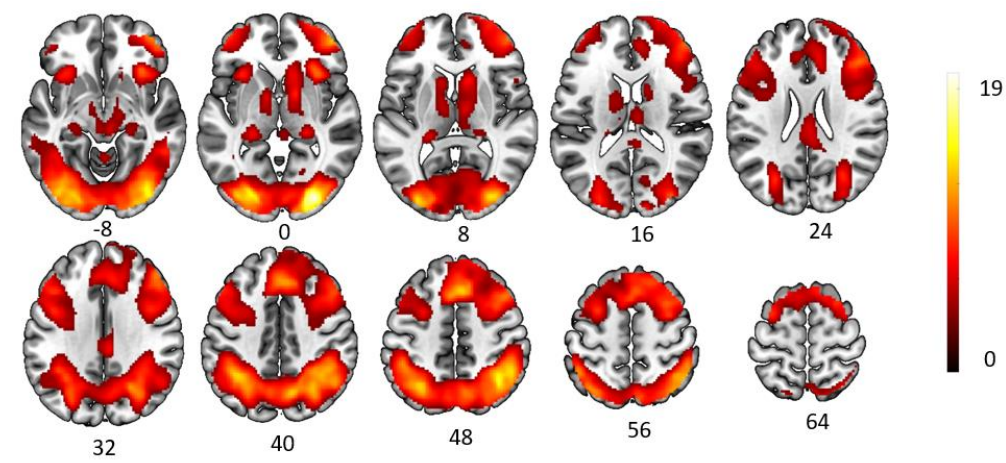

Figure for supplementary materials: Results whole-brain analysis inconsistent condition versus control condition during the feedback phase (cluster defining threshold of $\mathrm{p}<0.001$, cluster probability of $\mathrm{p}<0.05$, family wise error corrected). Activity changes are overlaid on an anatomical MNI template. Color bars indicates t-values. The numbers below the brain slices indicate z-coordinates. 
Trust behavior and friendships

Table for supplementary materials

Mean and standard deviation of investment per trial

\begin{tabular}{|c|c|c|c|c|}
\hline Trial number & $\begin{array}{l}\text { Mean investment } \\
\text { consistent } \\
\text { condition }\end{array}$ & $\begin{array}{l}\text { Standard deviation } \\
\text { investment in } \\
\text { consistent } \\
\text { condition }\end{array}$ & $\begin{array}{l}\text { Mean investment } \\
\text { inconsistent } \\
\text { condition }\end{array}$ & $\begin{array}{l}\text { Standard deviation } \\
\text { investment in } \\
\text { inconsistent } \\
\text { condition }\end{array}$ \\
\hline $\begin{array}{l}\text { Acquaintance } \\
\text { round } 1\end{array}$ & 5.26 & 2.16 & 5.3 & 2.03 \\
\hline $\begin{array}{l}\text { Acquaintance } \\
\text { round } 2\end{array}$ & 5.36 & 2.32 & 5.74 & 2.18 \\
\hline $\begin{array}{l}\text { Acquaintance } \\
\text { round } 3\end{array}$ & 5.92 & 2.37 & 5.62 & 2.17 \\
\hline 1 & 6.36 & 2.35 & 4.64 & 2.31 \\
\hline 2 & 6.54 & 2.56 & 5.04 & 2.3 \\
\hline 3 & 6.3 & 2.51 & 5.42 & 2.37 \\
\hline 4 & 6.46 & 2.31 & 5.40 & 2.38 \\
\hline 5 & 6.48 & 2.44 & 5.24 & 2.71 \\
\hline 6 & 7.18 & 2.12 & 6.06 & 2.51 \\
\hline 7 & 6.38 & 2.55 & 6.02 & 2.45 \\
\hline 8 & 6.44 & 2.94 & 5.74 & 2.71 \\
\hline 9 & 6.6 & 2.43 & 5.84 & 2.55 \\
\hline 10 & 6.1 & 2.38 & 6.18 & 2.83 \\
\hline 11 & 6.08 & 2.34 & 6.12 & 2.73 \\
\hline 12 & 6.48 & 2.51 & 5.76 & 2.58 \\
\hline 13 & 6.26 & 2.59 & 5.78 & 2.64 \\
\hline 14 & 6.9 & 2.34 & 5.94 & 2.82 \\
\hline
\end{tabular}


Trust behavior and friendships

\begin{tabular}{lllll}
15 & 6.04 & 2.99 & 5.78 & 2.87 \\
\hline 16 & 6.28 & 2.19 & 6.12 & 2.7 \\
\hline 17 & 6.12 & 2.78 & 5.94 & 2.82 \\
\hline 18 & 6.2 & 2.56 & 6.30 & 2.86 \\
\hline 19 & 6.54 & 2.75 & 6.28 & 2.62 \\
\hline 20 & 6.12 & 3.05 & 6.02 & \\
\hline
\end{tabular}


Trust behavior and friendships

Figure captions:

Figure 1. An experimental trial (left) and a control trial (right). Durations of the different events are indicated above the screenshots.

Figure 2. Significant three-way interaction between time (i.e. trials during the trust game), condition and age.

Figure 3. Significant three-way interaction between time (i.e. trials during the trust game), condition and eigencentrality. For visual purposes, eigencentrality is displayed using z-scores.

Figure 4. The left TPJ (left, $M=0.06, S D=0.22$ ) and the right dlPFC (right, $M=0.11, S D=0.39$ ) are the two regions of interest that show increased activity during the feedback phase of the inconsistent condition compared to consistent condition. 